\title{
G-Protein Beta3 Subunit C825T Polymorphism in Patients With Overlap Syndrome of Functional Dyspepsia and Irritable Bowel Syndrome
}

\author{
Han Gyeol Kim, Kwang Jae Lee, ${ }^{*}$ Sun Gyo Lim, Jae Yeon Jung and Sung Won Cho \\ Department of Gastroenterology, Ajou University School of Medicine, Suwon, Gyeonggi-do, Korea
}

\section{Background/Aims}

Guanine nucleotide binding protein (G-protein) beta polypeptide 3 (GNB3) C825T polymorphism alters intracellular signal transduction, which may lead to motor or sensory abnormalities of the gastrointestinal tract. The aim of the present study was to evaluate the association of the GNB3 C825T polymorphism with susceptibility to overlap syndrome of functional dyspepsia (FD) and irritable bowel syndrome (IBS) in a Korean population.

\section{Methods}

One hundred sixty-seven patients with FD alone, 60 patients with IBS alone, 85 patients with the overlap of FD and IBS, and 434 asymptomatic healthy subjects participated in the study. Genotyping for GNB3 C825T polymorphism was performed using their blood samples.

\section{Results}

No association of GNB3 genotypes in patients with FD alone, IBS alone or overlap phenotype, when compared to genotypes in controls, was detected. The frequency of $\mathrm{CT}$ and $\mathrm{TT}$ genotypes relative to the $\mathrm{CC}$ genotype for the phenotypes of FD alone, IBS alone and the coexistence of FD and IBS did not significantly differ. Comparison of the TT genotype with the CC/CT genotype showed no significant association for each phenotype group.

\section{Conclusions}

There is no apparent association of the GNB3 C825T polymorphism with the susceptibility to FD, IBS or the overlap of FD and IBS. Larger-scale studies and further investigation on other candidate genes are required.

\section{(J Neurogastroenterol Motil 2012;18:205-210)}

Key Words

Functional dyspepsia; G-protein beta3 subunit; Irritable bowel syndrome; Syndrome

Received: February 6, 2012 Revised: February 25, 2012 Accepted: March 8, 2012

(c) This is an Open Access article distributed under the terms of the Creative Commons Attribution Non-Commercial License (http://creativecommons. org/licenses/by-nc/3.0) which permits unrestricted non-commercial use, distribution, and reproduction in any medium, provided the original work is properly cited.

*Correspondence: Kwang Jae Lee, MD, PhD

Department of Gastroenterology, Ajou University School of Medicine, San 5 Wonchon-dong, Youngtong-gu, Suwon, Gyeonggi-do 443-721, Korea

Tel: +82-31-219-6939, Fax: +82-31-219-5999, E-mail: kj|@ajou.ac.kr

Financial support: This study was partially supported by a grant of the Korea Health 21 R\&D project, Ministry of Health, Welfare and Family Affairs, Republic of Korea (No. A010383).

Conflicts of interest: None. 


\section{Introduction}

Familial aggregation of patients with functional gastrointestinal disorders indicates that susceptibility to functional gastrointestinal disorders may be influenced by hereditary factors. ${ }^{1}$ Genetic variations of potential modulators of gastrointestinal sensory and motor function appear to influence the genesis of symptoms in patients with functional gastrointestinal disorders. There are twin studies that have shown that identical twins will have a greater concordance of having IBS than dizygotic twins, suggesting that genetic factors may contribute to the pathogenesis of IBS. $^{2}$ However, the susceptibility genes for functional gastrointestinal disorders are unknown yet and remain to be identified.

Guanine nucleotide binding protein (G-protein) beta polypeptide 3 (GNB3) gene is known to be associated with FD or IBS. A common $\mathrm{C} 825 \mathrm{~T}$ polymorphism has been described in the gene GNB3 that encodes the beta3 subunit of heterotrimeric G-proteins. This polymorphism gives rise to 3 possible genotypes $(\mathrm{C} / \mathrm{C}, \mathrm{T} / \mathrm{C}$ and $\mathrm{T} / \mathrm{T})$. The $825 \mathrm{~T}$ allele of the $\mathrm{T} / \mathrm{C}$ or $\mathrm{T} / \mathrm{T}$ genotype is associated with alternative splicing of the gene and the formation of a truncated but functionally active splice variant. Studies on the genetic variation associated with FD and IBS have produced inconsistent results, which is probably attributable to a complex multi-factorial pathophysiology and ethnic differences. For example, the $\mathrm{C} / \mathrm{C}$ genotype of $\mathrm{C} 825 \mathrm{~T}$ polymorphism is associated with unexplained dyspepsia in Germany. ${ }^{3}$ Whereas, homozygous $\mathrm{T}$ or $\mathrm{C}$ alleles of the GNB3 $\mathrm{C} 825 \mathrm{~T}$ polymorphism is associated with meal-unrelated dyspepsia in a United States (US) community. Tertiary referral FD in the Netherlands and dyspepsia in the Helicobacter pylori-negative Japanese population are reported to be associated with the $825 \mathrm{~T}$ allele of the GNB3 gene. ${ }^{5,6} \mathrm{GNB} 3 \mathrm{C} 825 \mathrm{~T}$ polymorphism is not associated with lower functional gastrointestinal disorders. ${ }^{7}$

The $825 \mathrm{~T}$ allele is believed to be associated with enhanced G-protein activation and, thereby, increased cellular or physiologic responses. ${ }^{8}$ Homozygous $825 \mathrm{C}$ allele carriers (C/C genotype) are characterized by diminished signal transduction responses. Evidence shows that the GNB3 status is associated with depression, ${ }^{9}$ increased immune cell activation ${ }^{10}$ and altered activation of $\alpha 2$-adrenoceptors. ${ }^{8} \mathrm{~A}$ diminished or increased signal transduction response could lead to motor or sensory abnormalities of the gastrointestinal tract that can be pathophysiologic mechanisms underlying FD and IBS. ${ }^{11}$ FD and IBS are highly prevalent diseases comprising $10 \%$ to $25 \%$ of the general popula- tion. ${ }^{12}$ These 2 conditions commonly coexist. ${ }^{13,14}$ Clinical studies regarding the pathophysiology of FD and IBS have identified a number of functional abnormalities including disturbances in gastrointestinal motor and sensory function. ${ }^{15-18}$ The symptom pattern and underlying pathophysiological mechanisms may be different between patients with FD alone compared to patients with both FD and IBS. It has been reported that patients with FD and IBS do not differ in the prevalence of delayed gastric emptying or of impaired gastric accommodation to a meal, but have a greater prevalence of hypersensitivity to gastric distension. ${ }^{19}$ Thus, we hypothesized that GNB3 C825T polymorphism, particularly $825 \mathrm{~T}$ allele, is more likely to be associated with the overlap of FD and IBS rather than FD alone or IBS alone. In the present study, we aimed to study the association of the GNB3 C825T polymorphism with susceptibility to FD alone, IBS alone and the overlap of FD and IBS in a Korean population.

\section{Materials and Methods}

\section{Study Subjects}

Patient cases and control subjects of the study were recruited between January 2007 and December 2008 from visitors to Department of Gastroenterology and the Health Promotion Center of Ajou University Hospital. Subjects without symptoms who visited for health check-up were recruited as healthy controls, and patients with recurrent abdominal symptoms were recruited as patient cases. They underwent examinations including endoscopy, excluded organic causes, and completed a self-administered questionnaire for the diagnosis of FD and IBS according to the ROME III criteria. ${ }^{20}$ All subjects were Koreans. One hundred sixty-seven patients with FD alone, 60 patients with IBS alone, 85 patients with the overlap of FD and IBS and 434 asymptomatic healthy subjects participated in the study. They allowed the use of their blood for the present study. This study was approved by the institutional review board of Ajou University Hospital and written informed consent was obtained from all participants.

\section{Questionnaire}

The questionnaire included information on demographics and Rome III criteria for the diagnosis of FD and IBS. Dyspeptic symptoms were defined as pain or discomfort in the upper abdomen for the last 3 months, with symptom onset at least 
6 months prior to the check-up. IBS was defined as recurrent abdominal pain or discomfort at least 3 days per month in the last 3 months associated with 2 or more of the following: improvement with defecation, onset associated with a change in stool frequency, or onset associated with a change in stool form.

\section{Genotyping}

The blood samples were stored at $-80^{\circ} \mathrm{C}$ until use. Genomic DNA was isolated from whole blood using G-DEX ${ }^{\mathrm{TM}}$ blood genomic DNA purification kits (Intron Biotechnology Inc., Seongnam, Korea) and quantified using the Picogreen dsDNA quantification reagent according to the standard protocol (Molecular Probes, Eugene, OR, USA). Genotyping was performed using TaqMan Pre-Designed single nucleotide polymorphism (SNP) Genotyping Assay (Applied Biosystems, Foster City, CA, USA). The allelic discrimination of SNPs on the basis of the TaqMan technology was performed. Fluorescence is released while the target fragment is amplified by the sequence detection primers. PCR is performed in standard PCR machines and in a real time PCR machine. This platform is particularly well suited for the analysis of a large number of samples with a moderate number of SNPs.

\section{Statistical Methods}

We presumed that a $10 \%$ increase in the prevalence of a genotype would be of clinical relevance. Assuming that a level of significance is $5 \%(\alpha=0.05)$ and a power is $80 \%(\beta=0.80)$, a sample of 329 patients and 329 healthy controls would be sufficient to reveal clinically relevant differences. PASS 2008 software (NCSS, Kaysville, Utah, USA) was used for the calculation of sample size. Deviations from the Hardy-Weinberg equilibrium were checked using chi-square tests. We compared GNB3 genotypes in patients with FD alone, IBS alone and the overlap of FD and IBS vs asymptomatic controls. The calculation of the odds ratio (OR), 95\% confidence intervals (CI) and $P$-values were performed using a multiple logistic regression model after controlling for age and gender as covariates. The $P$-value threshold for statistical significance used in this study was 0.05. SAS statistical software (SAS 9.1 and SAS Enterprise Guide 4.1; SAS Institute, Cary, NC, USA) was used for the statistical analysis. A $P<0.05$ was considered significant.

\section{Results}

Participant demographics and GNB3 C825T genotype distribution are summarized in Table 1 . No significant difference was found between the groups for gender and age. The GNB3 genotype distribution in all study subjects was $196 \mathrm{CC}(26.3 \%)$, 364 CT (48.8\%) and 186 TT (24.9\%). This distribution did not deviate from those expected under the Hardy-Weinberg equilibrium $(P>0.05)$. The distribution of allele and genotype frequencies did not differ significantly between males and females.

Table 2 shows the results of logistic regression analysis adjusted for age and sex on the association of genotype with each group, compared with asymptomatic controls. No association of genotype in subjects with FD alone, IBS alone or overlap phenotype compared to that in controls was detected $(P>0.05)$. The OR for the co-dominant, dominant and recessive models are shown in Table 2. The frequency of GNB3 C825T CT and TT genotypes relative to the $\mathrm{CC}$ genotype for the phenotypes of $\mathrm{FD}$ alone, IBS alone, and the overlap of FD and IBS did not significantly differ. Comparison of the TT genotype with the

Table 1. Demographics and Genotype Distribution in Subjects With Functional Dyspepsia Alone, Asymptomatic Controls, Irritable Bowel Syndrome Alone and the Overlap of Functional Dyspepsia and Irritable Bowel Syndrome

\begin{tabular}{|c|c|c|c|c|}
\hline & \multicolumn{4}{|c|}{ Group $^{a}$} \\
\hline & Controls & $\mathrm{FD}$ & IBS & Overlap \\
\hline Gender (M:F [n]) & $167: 267$ & $62: 105$ & $25: 35$ & $30: 55$ \\
\hline Mean age ( $\pm \mathrm{SD})$, yr & $47( \pm 15)$ & $49( \pm 15)$ & $43( \pm 12)$ & $44( \pm 15)$ \\
\hline \multicolumn{5}{|c|}{ GNB3 C825T Genotype (n [\%]) } \\
\hline $\mathrm{CC}$ & $112(25.8)$ & $52(31.1)$ & $16(26.7)$ & $16(18.8)$ \\
\hline $\mathrm{CT}$ & $215(49.5)$ & $76(45.5)$ & $31(51.7)$ & $42(49.4)$ \\
\hline TT & $107(24.7)$ & $39(23.4)$ & $13(21.7)$ & $27(31.8)$ \\
\hline Total & 434 & 167 & 60 & 85 \\
\hline
\end{tabular}

${ }^{a} \mathrm{FD}$ : functional dyspepsia alone, Controls: asymptomatic controls, IBS: irritable bowel syndrome alone, Overlap: coexistence of functional dyspepsia and irritable bowel syndrome.

GNB3, guanine nucleotide binding protein beta polypeptide 3 . 
Table 2. Logistic Regression Analysis Adjusted for Age and Sex in Subjects With Functional Dyspepsia Alone, Asymptomatic Controls, Irritable Bowel Syndrome Alone and the Overlap of Functional Dyspepsia and Irritable Bowel Syndrome

\begin{tabular}{|c|c|c|c|c|c|c|}
\hline \multirow{2}{*}{ Model } & \multicolumn{2}{|c|}{$\mathrm{FD}^{\mathrm{a}}$ vs Controls ${ }^{\mathrm{b}}$} & \multicolumn{2}{|c|}{ IBS $^{\mathrm{c}}$ vs Controls } & \multicolumn{2}{|c|}{ Overlap ${ }^{\mathrm{d}}$ vs Controls } \\
\hline & $P$-value & OR $(95 \% \mathrm{CI})$ & $P$-value & OR $(95 \% \mathrm{CI})$ & $P$-value & OR $(95 \% \mathrm{CI})$ \\
\hline Co-dominant & 0.329 & $1.13(0.88-1.46)$ & 0.723 & $0.93(0.63-1.37)$ & 0.112 & $1.31(0.94-1.83)$ \\
\hline Dominant & 0.718 & $1.04(0.84-1.28)$ & 0.671 & $0.93(0.67-1.29)$ & 0.160 & $1.21(0.93-1.55)$ \\
\hline Recessive & 0.220 & $1.13(0.93-1.38)$ & 0.874 & $0.97(0.72-1.33)$ & 0.234 & $1.19(0.89-1.61)$ \\
\hline MAF & 0.321 & $1.07(0.94-1.21)$ & 0.723 & $0.97(0.79-1.17)$ & 0.112 & $1.14(0.97-1.35)$ \\
\hline
\end{tabular}

${ }^{a} \mathrm{FD}$ : functional dyspepsia alone, ${ }^{\mathrm{b}}$ Controls: asymptomatic controls, ${ }^{\mathrm{c}} \mathrm{IBS}$ : irritable bowel syndrome alone, ${ }^{\mathrm{d}}$ Overlap: coexistence of functional dyspepsia and irritable bowel syndrome.

Co-dominant, CC vs CT vs TT; Dominant, CC vs CT and TT; Recessive, CC and CT vs TT; MAF, minor allele frequency.

CC/CT genotype showed no significant association for each phenotype group.

\section{Discussion}

This study evaluated GNB3 C825T polymorphism in a Korean population comprising people with FD alone, IBS alone, the overlap of FD and IBS, and healthy controls who visited outpatient clinic for abdominal symptoms or a health care unit for annual health check-up. We failed to demonstrate an association between GNB3 C825T polymorphism and the overlap of FD and IBS. Furthermore, no significant association between GNB3 C825 T polymorphism and the existence of FD alone or IBS alone was found in the present study. Our findings are consistent with a previous study from a Korean population. ${ }^{21}$ In contrast, data from Japan show that homozygous GNB3 $825 \mathrm{~T}$ is associated with Japanese dyspeptic subjects without H. pylori infection or with epigastric pain syndrome-like symptomatology. ${ }^{6,22}$ We explored whether any subgroup of FD or IBS is different from controls since the subgroups of these syndromes are likely to have different pathogenesis and may confound the results. However, we did not find any association of homozygous GNB3 $825 \mathrm{~T}$ with epigastric pain syndrome-like dyspepsia, postprandial distress syndrome-like dyspepsia, IBS with diarrhea or IBS with constipation (data not shown).

Similarly, in the West, conflicting results have been reported. While a study from the Netherlands demonstrated that $\mathrm{T}$ allele carriers of GNB3 C825 T polymorphism are associated with dyspepsia, ${ }^{5}$ a report in a German population shows that homozygous GNB3 825C status is associated with unexplained dyspepsia. ${ }^{3}$ Furthermore, reports from the US revealed that meal-unrelated dyspepsia is associated with both the homozygous GNB3 825 T and $\mathrm{C}$ genotypes. ${ }^{4}$ These contrasting observations may be parti- ally explained by a racial difference and heterogeneity of the disease. In addition, the diagnostic criteria of FD, sample selection or sample size may affect the outcome. $\mathrm{T}$ alleles of the GNB3 C825T polymorphism are reported to be more strongly correlated with IBS with constipation in the Korean population. ${ }^{23}$ The study population included 88 control cases and 12 constipation predominant IBS cases. This sample size does not appear to be adequate for the study on the association of GNB3 C825T polymorphism with disease susceptibility. Moreover, there is a contradictory study showing that GNB3 C825T polymorphism is not associated with FD and IBS in Koreans. ${ }^{21}$ The study population consisted of 70 healthy controls, 62 FD patients and 49 IBS patients. The number of this study population appears to be too small to show the association of genetic polymorphisms with disease susceptibility. Relatively small sample sizes may produce the type II error. The sample size of the present study is greater than that of the previous Korean studies. Based on the results of the sample size calculation that 224 patients and 448 healthy controls were required, we recruited 312 patients and 434 healthy controls in the present study. However, the number of each group, particularly the number of each subgroup, might be not sufficient.

Disturbed gut sensory or motor function, dysfunction of the autonomic nervous system, and underlying psychiatric disturbance have been suggested to contribute to the pathophysiology of functional gastrointestinal disorders like FD and IBS. ${ }^{15-19}$ All of these abnormalities may be associated with the alteration of intracellular signal transduction. Approximately up to $80 \%$ of all known membrane receptors that are linked to intracellular effector systems are coupled to G-proteins. ${ }^{8,10} \mathrm{G}$-proteins play a crucial role in intracellular signal transduction. Therefore, qualitative or quantitative changes in G-proteins may lead to functional changes by inhibiting or enhancing intracellular signal 
transduction. The GNB3 gene encodes the $\beta 3$ subunit of heterotrimeric G-proteins, which is known to potentially affect intracellular signal transduction and biological activity, and the $825 \mathrm{~T}$ is associated with enhanced G-protein activation. ${ }^{24}$ Given that patients with FD and IBS do not differ in the prevalence of delayed gastric emptying or of impaired gastric accommodation to a meal, but have a greater prevalence of hypersensitivity to gastric distension, ${ }^{19}$ GNB3 C825T polymorphism, particularly $825 \mathrm{~T}$ allele, is more likely to be associated with the overlap of FD and IBS rather than FD alone or IBS alone. It is conceivable that the GNB3 genotype is involved in the generation of common pathophysiologic mechanisms underlying FD and IBS. Transition of functional gastrointestinal disorders in their natural history supports our hypothesis. To my knowledge, this is the first study investigating the genetic factors of overlap syndrome. However, in the present study, we failed to find the association of the GNB3 polymorphism with the overlap of FD and IBS. Genetic polymorphisms of other candidate genes warrant further investigation.

The data in the present study are from outpatient clinic of the university hospital and from subjects undergoing health checkup. We selected subjects who underwent upper or lower gastrointestinal endoscopy in order to exclude organic causes. Therefore, uninvestigated subjects were not included in the study. All subjects are Korean. Korean population is known to be genetically homogenous. Although GNB3 C825T polymorphism does not have any statistically significant association with FD and IBS in the present study, this negative association might be affected by multiple pathophysiologic mechanisms underlying functional gastrointestinal disorders.

In conclusion, our findings suggest that there is no apparent association of the GNB3 C825T polymorphism with the susceptibility to FD, IBS or the overlap of FD and IBS. Larger-scale studies and further investigation on other candidate genes are required.

\section{References}

1. Locke GR 3rd, Zinsmeister AR, Talley NJ, Fett SL, Melton LJ 3rd. Familial association in adults with functional gastrointestinal disorders. Mayo Clin Proc 2000;75:907-912.

2. Bengtson MB, Rønning T, Vatn MH, Harris JR. Irritable bowel syndrome in twins: genes and environment. Gut 2006;5 5:1754-1759.

3. Holtmann G, Siffert W, Haag S, et al. G-protein $\beta 3$ subunit 825 CC genotype is associated with unexplained (functional) dyspepsia. Gastroenterology 2004;126:971-979.

4. Camilleri CE, Carlson PJ, Camilleri M, et al. A study of candidate genotypes associated with dyspepsia in a U.S. community. Am J Gastroenterol 2006;101:581-592.

5. van Lelyveld N, Linde JT, Schipper M, Samsom M. Candidate genotypes associated with functional dyspepsia. Neurogastroenterol Motil 2008;20:767-773.

6. Tahara T, Arisawa T, Shibata $\mathrm{T}$, et al. Homozygous $825 \mathrm{~T}$ allele of the GNB3 protein influences the susceptibility of Japanese to dyspepsia. Dig Dis Sci 2008;53:642-646.

7. Andresen V, Camilleri M, Kim HJ, et al. Is there an association between GNB3-C825T genotype and lower functional gastrointestinal disorders? Gastroenterology 2006;130:1985-1994.

8. Baumgart D, Naber C, Haude M, et al. Protein beta3 subunit $825 \mathrm{~T}$ allele and enhanced coronary vasoconstriction on alpha2-adrenoceptor activation. Circ Res 1999;85:965-969.

9. Zill P, Baghai TC, Zwanzger P, et al. Evidence for an association between a G-protein beta3-gene variant with depression and response to antidepressant treatment. Neuroreport 2000;11:1893-1897.

10. Lindemaann $M$, Virchow $S$, Ramann $F$, et al. The $G$ protein beta3 subunit $825 \mathrm{~T}$ allele is a genetic marker for enhanced $\mathrm{T}$ cell response. FEBS Lett 2001;495:82-86.

11. Holtmann G, Gschossmann J, Neufang-Hüber J, Gerken G, Talley NJ. Differences in gastric mechnosensory function after repeated ramp distensions in non-consulters with dyspepsia and healthy controls. Gut 2000;47:332-336.

12. Locke GR 3rd. The epidemiology of functional gastrointestinal disorders in North America. Gastroenterol Clin North Am 1996;25:119.

13. Agréus L, Svärdsudd K, Nyrén O, Tibblin G. Irritable bowel syndrome and dyspepsia in the general population: overlap and lack of stability over time. Gastroenterology 1995;109:671-680.

14. Caballero-Plasencia AM, Sofos-Kontoyannis S, Valenzuela-Barraco M, Martín-Ruiz JL, Casado-Caballero FJ, López-Mañas JG. Irritable bowel syndrome in patients with dyspepsia: a community-based study in southern Europe. Eur J Gastroenterol Hepatol 1999;11:517-522.

15. Stanghellini V, Tosetti C, Paternico A, et al. Risk indicators of delayed gastric emptying of solids in patients with functional dyspepsia. Gastroenterology 1996;110:1036-1042.

16. Tack J, Piessevaux H, Coulie B, Caenepeel P, Janssens J. Role of impaired gastric accommodation to a meal in functional dyspepsia. Gastroenterology 1998;115:1346-1352.

17. Holtmann G, Goebell H, Jockenhovel F, Talley NJ. Altered vagal and intestinal mechano-sensory function in chronic unexplained dyspepsia. Gut 1998;42:501-506.

18. Coffin B, Azpiroz F, Guarner F, Malagelada JR. Selective gastric hypersensitivity and reflex hyporeactivity in functional dyspepsia. Gastroenterology 1994;107:1345-1351.

19. Corsetti M, Caenepeel P, Fischler B, Janssens J, Tack J. Impact of coexisting irritable bowel syndrome on symptoms and pathophysiological mechanisms in functional dyspepsia. Am J Gastroenterol 2004;99:1152-1159.

20. Tack J, Talley NJ, Camilleri M, et al. Functional gastroduodenal disorders. Gastroenterology 2006;130:1466-1479.

21. Park HY, Jahng JH, Lee YJ, Park H, Lee SI. Serotonin Transporter Gene and G-protein $\beta 3$ C825T Gene Polymorphism in Patients with Functional Dyspepsia and Irritable Bowel Syndrome. Kor J 
Neurogastroenterol Motil 2009;15:58-64.

22. Oshima T, Nakajima S, Yokoyama T, et al. The G-protein beta3 subunit 825 TT genotype is associated with epigastric pain syndrome-like dyspepsia. BMC Med Genet 2010;11:13.

23. Lee HJ, Lee SY, Choi JE, et al. G protein beta3 subunit, interleukin-10, and tumor necrosis factor-alpha gene polymorphisms in
Koreans with irritable bowel syndrome. Neurogastroenterol Motil 2010;22:758-763.

24. Siffert W, Rosskopf D, Siffert G, et al. Association of a human Gprotein beta3 subunit variant with hypertension. Nat Genet 1998;18: $45-48$. 